\section{(6) OPEN ACCESS}

\title{
Characterisation of faecal protease activity in irritable bowel syndrome with diarrhoea: origin and effect of gut transit
}

\author{
David Tooth, ${ }^{1}$ Klara Garsed, ${ }^{2}$ Gulzar Singh, ${ }^{2}$ Luca Marciani, ${ }^{2}$ Ching Lam, ${ }^{2}$ \\ Imogen Fordham, ${ }^{2}$ Annie Fields, ${ }^{2}$ Rawinder Banwait, ${ }^{2}$ Melanie Lingaya, ${ }^{2}$ \\ Robert Layfield, ${ }^{1}$ Maggie Hastings, ${ }^{3}$ Peter Whorwell, ${ }^{3}$ Robin Spiller ${ }^{2}$
}

\begin{abstract}
- Additional material is published online only. To view please visit the journal online (http://dx.doi.org/10.1136/ gutjnl-2012-304042).

${ }^{1}$ School of Biomedical Sciences, University of Nottingham, Nottingham, Nottinghamshire, UK ${ }^{2}$ Nottingham Digestive Diseases NHIR BRU, University of Nottingham, Nottingham, Nottinghamshire, UK ${ }^{3}$ Department of

Gastroenterology, University of South Manchester,

Wythenshawe Hospital, Manchester, Lancashire, UK
\end{abstract}

\section{Correspondence to} Professor Robin Spiller, NIHR Nottingham Digestive Diseases Biomedical Research Unit, Nottingham Digestive Diseases Centre, University of Nottingham, University Hospital Nottingham, Queen's Medical Centre, E Floor West Block, Nottingham, Nottinghamshire NG7 2UH, UK;

robin.spiller@nottingham.ac.uk

Received 31 October 2012

Revised 2 July 2013

Accepted 4 July 2013

Published Online First

2 August 2013

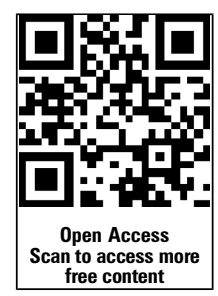

To cite: Tooth D, Garsed K, Singh G, et al. Gut 2014;63:753-760.

\section{ABSTRACT}

Objectives Faecal serine proteases (FSPs) may play a role in irritable bowel syndrome with diarrhoea (IBS-D), but their origin is unclear. We aimed to structurally characterise them and define the impact of colonic cleansing and transit time.

Design Faecal samples were obtained from 30 healthy volunteers (HV) and 79 patients with IBS-D participating in a trial of ondansetron versus placebo. Colonic transit was measured using radio-opaque markers. Samples were also obtained from $24 \mathrm{HV}$ before and after colonic cleansing with the osmotic laxative MoviPrep. FSPs were purified from faecal extracts using benzamidineSepharose affinity chromatography. SDS-PAGE profiled components were identified using trypsinolysis and tandem mass spectrometry. Functional protease activity in faecal extracts was measured using a colorimetric assay based on the proteolysis of azo-casein.

Results Protein analysis identified the most abundant FSPs as being of human origin and probably derived from pancreatic juice. Functional assays showed increased faecal protease (FP) and amylase in patients with IBS-D compared with HV. Those with higher amylase had significantly higher FP and greater anxiety. FP activity correlated negatively with whole gut transit in patients with IBS-D (Spearman $r=-0.32, p=0.005$ ) and $\mathrm{HV}(r=-0.55, p=0.014)$. Colon cleansing caused $a$ significant rise in FP activity in HV from a baseline of median (IQR) 253 (140-426) to 1031 (435-2296), levels similar to those seen in patients with IBS-D. FSP activity correlated positively with days/week with urgency.

Conclusions The most abundant FSPs are of human origin. Rapid transit through the colon and/or decreased (possibly bacterial) proteolytic degradation increases their faecal concentration and could contribute to visceral hypersensitivity in patients with IBS-D. ClinicalTrials.gov NCT00745004.

\section{BACKGROUND}

Irritable bowel syndrome (IBS) is a ubiquitous condition characterised by abdominal pain or discomfort and altered bowel habit, reported by up to $10 \%$ of the population. ${ }^{1}$ The cause is essentially unknown, but several subtypes are recognised based on differing stool patterns including IBS with diarrhoea (IBS-D), IBS with constipation (IBS-C) and a mixed bowel habit (IBS-M) where stool

\section{Significance of this study}

What is already known about this subject?

- Patients with irritable bowel syndrome and diarrhoea (IBS-D) have increased faecal serine protease activity which may contribute to colonic hypersensitivity.

- The origin of the faecal proteases is unclear.

What are the new findings?

- Protein characterisation suggests the most abundant faecal serine proteases are human and probably pancreatic in origin.

- Bowel cleansing increases faecal protease activity, suggesting that bacteria degrade endogenous pancreatic proteases.

- Faecal protease activity is positively correlated with days/week with urgency and negatively correlated with colonic transit.

How might this impact on clinical practice in the foreseeable future?

- Increased faecal protease activity could exacerbate IBS-D symptoms by sensitising the rectum to distension and could be a new therapeutic target for the treatment of IBS-D.

consistency varies erratically. The precise cause of the disturbed bowel habit is unknown, but there has been much interest in recent reports that the faeces of patients with IBS-D have increased faecal serine protease (FSP) activity. ${ }^{2}$ These proteases have been shown to be the active component of faecal supernatants from patients with IBS-D which, when instilled into the mouse colon, act via PAR-2 receptors to cause increased permeability, mucosal inflammation and visceral hypersensitivity-characteristic features of IBS. ${ }^{3}$ These FSPs have so far only been assessed using functional assays and have not been structurally identified, so their origin is unclear. It has been shown that they are also increased in patients with ulcerative colitis but not in IBS-C or IBS-M, healthy controls, nor individuals with acute infectious diarrhoea-facts which has been used to argue that accelerated transit was not the cause of increased serine protease activity. 
Previous work showed that serine proteases were unlikely to be of mast cell origin since mast cell tryptase was not increased, ${ }^{4}$ nor were they thought to be of neutrophil origin since neutrophil markers such as myeloperoxidase ${ }^{4}$ or calprotectin ${ }^{5}$ are not elevated in IBS-D. We hypothesised that these FSPs are likely to be of bacterial origin. Inhibiting these with protease inhibitors can reverse the colonic hypersensitivity induced in animal models, suggesting that reducing faecal proteases (FPs) might be a therapeutic target in patients with IBS. ${ }^{2}$

The aim of this study was therefore to characterise FSPs both structurally and functionally in patients with IBS and healthy controls to determine their origin as a first step to devising a therapeutic intervention to reduce visceral hypersensitivity in IBS. Our initial protein sequencing studies ${ }^{6}$ suggested that the enzyme activity was likely to be of human origin. We hypothesised that increased FP activity might be due to accelerated transit and/or altered faecal microbiota reducing bacterial degradation which normally accounts for the 20-fold fall in protease activity in human faeces compared with ileostomy output. ${ }^{7}$ We tested this hypothesis indirectly by examining the correlation of total FP activity with specific immunoassays for pancreatic elastase and pancreatic $\alpha$-amylase and also with whole gut transit. Finally, we accelerated transit and reduced faecal microbiota by using an osmotic laxative polyethylene glycol and showed that this increased FP activity.

\section{METHODS}

The study consisted of two parts. Part 1 was descriptive and method development and involved the first 36 patients with IBS-D entering a randomised placebo controlled trial of ondansetron and nine healthy controls. Since our studies showed a possible effect of colonic transit on faecal levels of another pancreatic enzyme (amylase), Part $2 \mathrm{a}$ involved assessment of the correlation with transit in 79 patients with IBS-D while Part $2 b$ examined the effect of colonic cleansing using an osmotic laxative MoviPrep in 24 healthy volunteers.

\section{Participants}

Healthy controls were recruited by advertisement and completed a Rome III questionnaire to exclude a diagnosis of IBS. All denied abdominal pain or discomfort.

Patients with IBS had already completed a full negative evaluation for other diseases in the Nottingham University Hospital NHS Trust gastroenterology outpatient clinic prior to entry into a randomised placebo-controlled crossover trial of ondansetron in 2009-2011, the results of which have been presented in abstract form ${ }^{8}$ and will be published separately.

Patients were aged 18-75 years and met Rome III criteria for IBS-D with at least $25 \%$ of the stools having a stool form score of 6 or 7 on the Bristol Stool Form Score (BSFS) and no more than $25 \%$ with a stool form score of 1 or 2 . Patients completed a stool diary during a 2 -week run-in period to confirm they met criteria before enrolment. They also completed the Hospital Anxiety and Depression Score and the Rome III questionnaire. Patients were excluded if they had had abdominal surgery other than appendicectomy and cholecystectomy or were unable to stop antidiarrhoeal drugs. The evaluation included a detailed history, examination, sigmoidoscopy and biopsy, full blood count, haematinics, electrolytes, tissue transglutaminase antibody, thyroid function, calcium, liver function tests as well as colonoscopy and biopsy to exclude microscopic colitis. Bile salt retention testing (selenium-75 homocholic acid taurine, SeHCAT) was performed where there was high-volume diarrhoea or nocturnal symptoms. Patients were instructed to drink one pint of skimmed milk after an overnight fast to screen for lactose intolerance. Those who developed symptoms were then tested with a formal breath hydrogen $50 \mathrm{~g}$ lactose tolerance test and those whose symptoms responded to a lactose-free diet were excluded.

\section{Study design}

The study comprised two parts. During Part 1 we structurally characterised the components detected in the selectively enriched serine protease fraction and assessed total FP activity as well as performing independent immunoassay of other pancreatic enzymes (ie, elastase and amylase) in a subgroup of the patients with IBS $(n=36)$ using their baseline samples before they received any treatment. These studies suggested that transit influenced faecal levels of at least one pancreatic enzyme (amylase), so in Part 2 we correlated FP with transit which were both measured after 5 weeks of placebo in the whole IBS-D patient cohort $(n=79)$ (Study 2a). Furthermore, since previous studies have suggested that bacterial degradation reduced FP, we examined the effect of bowel cleansing in 24 healthy controls (Study 2b).

\section{Part 1}

Healthy controls $(n=9)$ were asked to complete a 1-week stool diary documenting stool form using the BSFS, stool frequency and bowel symptoms including bloating, abdominal pain and urgency on a daily basis. They also provided a stool sample. Thirty-six patients with IBS-D entering our ondansetron RCT provided identical information as part of their baseline data before commencing the trial. The demographic characteristics of both groups are shown in table 1 .

\section{Part 2a: Effect of ondansetron}

The RCT of ondansetron was a 5-week crossover study with a 2-3-week washout period to ensure return to previous bowel habit. We used dose titration to optimise the response and avoid excessive constipation. Doses used ranged from $4 \mathrm{mg}$ every other day to $8 \mathrm{mg}$ three times daily. The final dose was maintained unchanged for the final 2 weeks when colonic transit was measured. On day 4 of the transit measurement the stool sample was provided to be analysed for FP for correlation with transit. Here we report only the values of FP obtained in the first 36 patients at baseline and all 79 patients who provided a suitable sample and had colonic transit measured during the placebo arm of the study. A total of 128 patients completed the multicentre trial but lack of stool sample (17) at the appropriate time and/or lack of valid transit measurement (29) meant that

Table 1 Subject demographics

\begin{tabular}{|c|c|c|c|c|c|c|}
\hline & $\begin{array}{l}\text { Patient } \\
\text { group }\end{array}$ & $\mathrm{N}$ & $\begin{array}{l}\text { Gender } \\
\text { M/F }\end{array}$ & $\begin{array}{l}\text { Age years } \\
\text { Mean } \\
\text { (SEM) }\end{array}$ & $\begin{array}{l}\text { Bowel } \\
\text { movements/day } \\
\text { Median (IQR) }\end{array}$ & $\begin{array}{l}\text { Anxiety } \\
\text { HADS } \\
\text { Mean (SEM) }\end{array}$ \\
\hline \multirow[t]{2}{*}{$\begin{array}{l}\text { Part } \\
1\end{array}$} & $\begin{array}{l}\text { Healthy } \\
\text { volunteers }\end{array}$ & 9 & $3 / 6$ & $25.4(2.3)$ & $1.4(0.7-2.0)$ & ND \\
\hline & IBS-D & 36 & $11 / 25$ & $39(2)$ & $2.8(2.1-4.2)$ & $9.0(0.8)$ \\
\hline \multirow[t]{2}{*}{$\begin{array}{l}\text { Part } \\
2 a\end{array}$} & $\begin{array}{l}\text { Healthy } \\
\text { volunteers }\end{array}$ & 21 & $5 / 16$ & $42.8(4)$ & $1.1(1-1.4)$ & $5.2(0.6)$ \\
\hline & IBS-D & 79 & $22 / 57$ & $41(2)$ & $2.7(2-3.9)$ & $9.2(0.6)$ \\
\hline $\begin{array}{l}\text { Part } \\
2 \mathrm{~b}\end{array}$ & $\begin{array}{l}\text { Healthy } \\
\text { volunteers }\end{array}$ & 24 & $12 / 12$ & $26.5(3)$ & $1.3(1.1-1.5)$ & $3.5(0.7)$ \\
\hline
\end{tabular}


here we analyse a subset of 79 with complete data. There was, however, no significant difference in demographics and symptom severity from those who did not provide complete data (see online supplementary table S1 appendix), so the subjects whose data are presented here are representative of the study group as a whole. We also recruited an additional 21 healthy controls, attempting to age and sex match our patient group. They completed a 1-week stool diary documenting bowel frequency, stool form of each stool using the BSFS, and whether there was abdominal pain or urgency for each day. They also completed the same questionnaires as those used in the RCT and underwent the same whole gut transit measurement. Demographic details are shown in table 1.

\section{Part 2b: Effect of osmotic laxative MoviPrep}

This was part of an imaging study demonstrating the increase in small bowel and colonic water after MoviPrep ingestion. ${ }^{9}$ As part of that study we collected stool samples to assess bacterial numbers and FP levels. Stool samples were collected using our standard protocol outlined below on the day before and 2, 14 and 28 days after purging with either a single dose of $2 \mathrm{~L}$ MoviPrep or two $1 \mathrm{~L}$ doses taken $12 \mathrm{~h}$ apart. Subjects ate a low residue diet the night before each study. This induced pronounced loose watery stools in both groups and, as expected, significantly reduced bacterial abundance.

\section{Stool collection}

Stool samples were collected at home by all subjects using three separate pots (Sterilin UK) on the morning of the visit and were delivered to the research centre within $2 \mathrm{~h}$ of defaecation when they were immediately frozen at $-70 \mathrm{C}^{\circ}$. Quantitative PCR using a universal bacterial probe was used to assess total bacterial count/abundance.

\section{Measurement of whole gut transit}

Transit was measured using the Metcalf method. ${ }^{10}$ Patients ingested 20 radio-opaque markers on the morning of days 1-3 and had a plain abdominal $\mathrm{x}$-ray on day 4 to count the number of pellets remaining. Transit was calculated in hours from the formula $1.2 \times$ number of remaining pellets as validated by Metcalf. $^{10}$

\section{Structural characterisation of FSPs}

We characterised the FSPs using the workflow outlined in figure 1.

Extraction and stabilisation of proteins from faecal samples

Samples $(1 \mathrm{~g})$ of stool stored at $-70^{\circ} \mathrm{C}$ were thawed and mixed in $5 \mathrm{~mL} 50 \mathrm{mM}$ Tris buffer, $150 \mathrm{mM} \mathrm{NaCl}, \mathrm{pH} 7.2$. Turbid suspensions were clarified using a sequential combination of centrifugation (10 min, $3000 \mathrm{RCF})$ and filtration $(0.2 \mu \mathrm{m})$. Particulate free supernatants were archived $\left(-70^{\circ} \mathrm{C}\right)$ until required for various assays or protein characterisation procedures.

\section{Quantitation of total protein}

Total protein quantitation was performed using the Bradford method $^{11}$ modified for low volume and high throughput. Briefly, equal volumes of sample and reagent were mixed in 96-well microplates and, following $20 \mathrm{~min}$ incubation, absorbance measurements at $595 \mathrm{~nm}$ were used to quantitate by reference to bovine serum albumin calibrants.

\section{Quantitation of total protease activity}

Total FP quantitation was performed using the non-specific proteolysis of azo-casein. The endoproteolysis of this liberates azo-peptides into the supernatant which are quantitated by absorbance measurement at $440 \mathrm{~nm}$ subsequent to protein precipitation with trichloroacetic acid. Protease activity was quantitated against bovine trypsin calibrant and expressed as trypsin units. Protein concentrations ranged from 0.08 to $1.7 \mathrm{mg} / \mathrm{mL}$
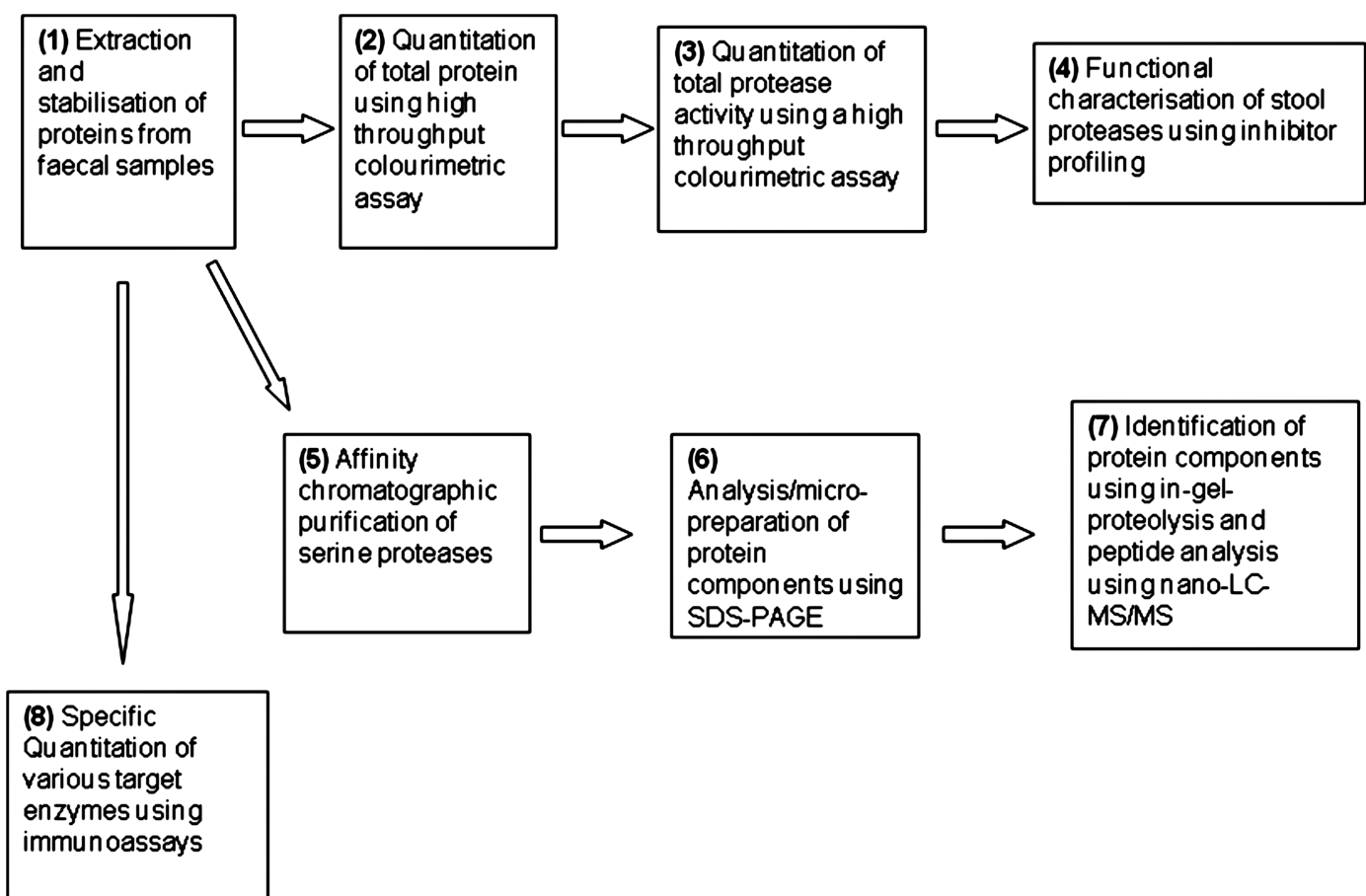

Figure 1 Methods and workflow. 
and measured protease activities were normalised against these values and expressed as units of trypsin/mg protein.

The protocol was essentially as described by Roka ${ }^{4}$ but was optimised to a high-throughput assay by application of 96-well microplates for extraction, incubation, clarification and absorbance plate reading and employing serial dilutions of test samples and calibrant. This enabled multiple positive and negative controls, calibration standards and samples at multiple dilutions and replicates to be rapidly processed. The serine protease inhibitor aprotinin was added to trypsin standards and faecal extracts at a range of concentrations and showed a concentration-dependent inhibition. At the higher dose of $100 \mu \mathrm{g} / \mathrm{mL}$ aprotinin, all FP from patients with IBS-D was inhibited $(n=7)$, suggesting most $\mathrm{FP}$ is in fact FSP.

\section{Affinity chromatographic purification of serine proteases}

We purified FSP by passing $0.45-0.55 \mathrm{mg}$ total protein in faecal extracts through $0.25 \mathrm{~mL}$ benzamidine-Sepharose resin (GE Healthcare, Amersham, UK) which specifically retains serine proteases in centrifugal spin columns. Samples were loaded and washed in $0.5 \mathrm{~mL} 50 \mathrm{mM}$ Tris, $\mathrm{pH}$ 7.4, $0.5 \mathrm{M}$ sodium chloride buffer and retained components were eluted using $0.5 \mathrm{~mL}$ $50 \mathrm{mM}$ glycine $\mathrm{pH} 3.0$ buffer. Proteins in eluate fractions were desalted using reversed phase solid-phase extraction essentially according to published procedures ${ }^{12}$ and characterised subsequently by SDS-PAGE profiling.

\section{Protein electrophoresis}

SDS-polyacrylamide gels (4-12\% Bis-Tris using MES buffer, Invitrogen, Paisley, UK) were electrophoresed under thiolreducing conditions according to vendor guidelines. Protein components were visualised by chemical staining using colloidal Coomassie Brilliant Blue G-250 (Pierce, Gelcode Blue) according to vendor guidelines.

\section{Identification of protein components}

Protein components in gel lanes were excised, reduced using dithiothreitol, alkylated using iodoacetamide and proteolysed using (Promega sequencing grade) porcine trypsin in $0.1 \mathrm{M}$ ammonium bicarbonate, $\mathrm{pH}$ 8.0. All protocols were standard procedures essentially according to Hellman et al. ${ }^{13}$

Proteolytic peptides were extracted, captured and desalted to a chromatographic trap (Dionex C18, $0.3 \times 5 \mathrm{~mm}$ ) and subjected to microcapillary high-pressure liquid chromatography (Phenomenex Jupiter Proteo $4 \mu \mathrm{m}, 0.075 \times 150 \mathrm{~mm}$ ) with tandem mass spectrometry (nano-LC-MS/MS) using a hybrid quadrupole time-of-flight (Waters Q-TOF2) instrument equipped with a nano-electrospray ion source in positive ion mode and calibrated against synthetic peptide product ions. Data-dependent product ion spectra were acquired 'on-the-fly' during test experiments. The MASCOT server (http://www. matrixscience.com) was used to interrogate the SWISS-PROT databases using total pooled precursor and product ion data and reports were compiled together with total molecular weight search (MOWSE) probability scores.

Assessment of faecal amylase and elastase using immunoassays Since our proteomic analysis suggested that a major serine protease component was trypsin, we decided also to assay pancreatic amylase and elastase. While trypsin and amylase are degraded by faecal bacteria and so normally excreted in only small amounts, elastase is more stable and readily detectable in stool and used widely to detect pancreatic insufficiency. Proprietary ELISA kit products were used to quantitate these other pancreatic enzymes in stool extracts-namely, Pancreatic Amylase ELISA (Biosupply, Bradford, UK) and elastase (ScheBo Pancreatic Elastase-1 Stool Test, Giessen, Germany).

In brief, the Pancreatic Amylase sandwich ELISA is performed by incubating the sample with immobilised mouse monoclonal antibodies to human pancreatic amylase. After washing these are incubated with a second anti-pancreatic amylase antibody linked to peroxidase and the amount bound assessed from the colour developed with the substrate tetramethylbenzidine. The ScheBo Pancreatic Elastase-1 Stool Test similarly uses an immobilised monoclonal antibody to human pancreatic elastase to bind the elastase. After incubation with a second biotinylated antibody, the bound antigen-antibody complex is detected by incubation with peroxidase/streptavidin and peroxidase activity is detected using the chromogen 2,2'-azoni-bis-3-ethylbenzothiazolin-6sulphonic acid and assessing the spectroscopic absorbance at $405 \mathrm{~nm}$. The intra-assay coefficient variation of repeated measures (CV) for elastase and amylase was 5.3\% and 8.9\%, respectively, and inter-assay $\mathrm{CV}$ was $14 \%$ and $11 \%$, respectively. A fluorescence reporter assay system (PHADIA-CAP, Phadia, Uppsala, Sweden) was also used to specifically quantitate mast cell tryptase. The assay was found to give undetectable readings when samples were spiked with human pancreatic trypsin (data not shown). The limit of detection was $1 \mu \mathrm{g} / \mathrm{L}$.

\section{Statistical analysis}

Values were assessed for normality using the Shapiro-Wilks statistic and the results are expressed as mean (SEM) for normally distributed data or median (IQR) if not normally distributed. Differences in FP activity between controls and patients with IBS-D were assessed using the Mann-Whitney U test while faecal elastase differences were assessed using the Student $t$ test. Differences between visits for the MoviPrep study were assessed using repeated measures ANOVA while the correlation between transit and FP activity was assessed using the non-parametric Spearman correlation test.

Correlations between FP activity and symptoms were assessed initially using the Spearman non-parametric test and then logistic regression was performed to predict values of FP above or below the mean value of 467 trypsin units/mg protein. The four variables shown to have significant correlations with FP levels were entered into a backwards conditional logistic regression model.

\section{Power calculations}

The initial studies were exploratory and so no power calculation could be performed. The ondansetron and MoviPrep trials were powered to show a change in stool consistency and changes in small bowel water content, respectively, while the faecal enzymes were secondary endpoints. However, the effect size of the MoviPrep purge was very substantial and we had a $90 \%$ power to detect a change of -101 trypsin units/mg, very much smaller than actually observed (778 trypsin units/mg).

\section{RESULTS}

\section{Selective purification of serine proteases from faecal supernatant}

Using a test mixture of bovine trypsinogen and equine myoglobin, we were able to demonstrate high selectivity of the benzamidine-Sepharose resin (see online supplementary appendix figure S1). When chromatographed, buffered faecal extracts from patients with IBS and controls similarly yielded retained components that, when profiled using micropreparative SDS-PAGE, yielded heterogeneous mixtures of components 
Healthy volunteers IBS-D Patients

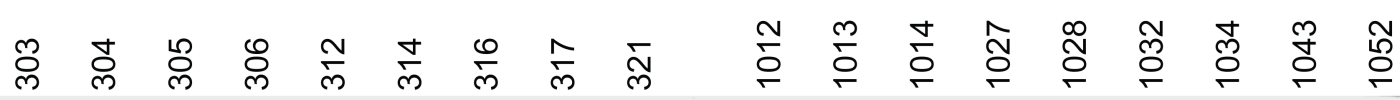

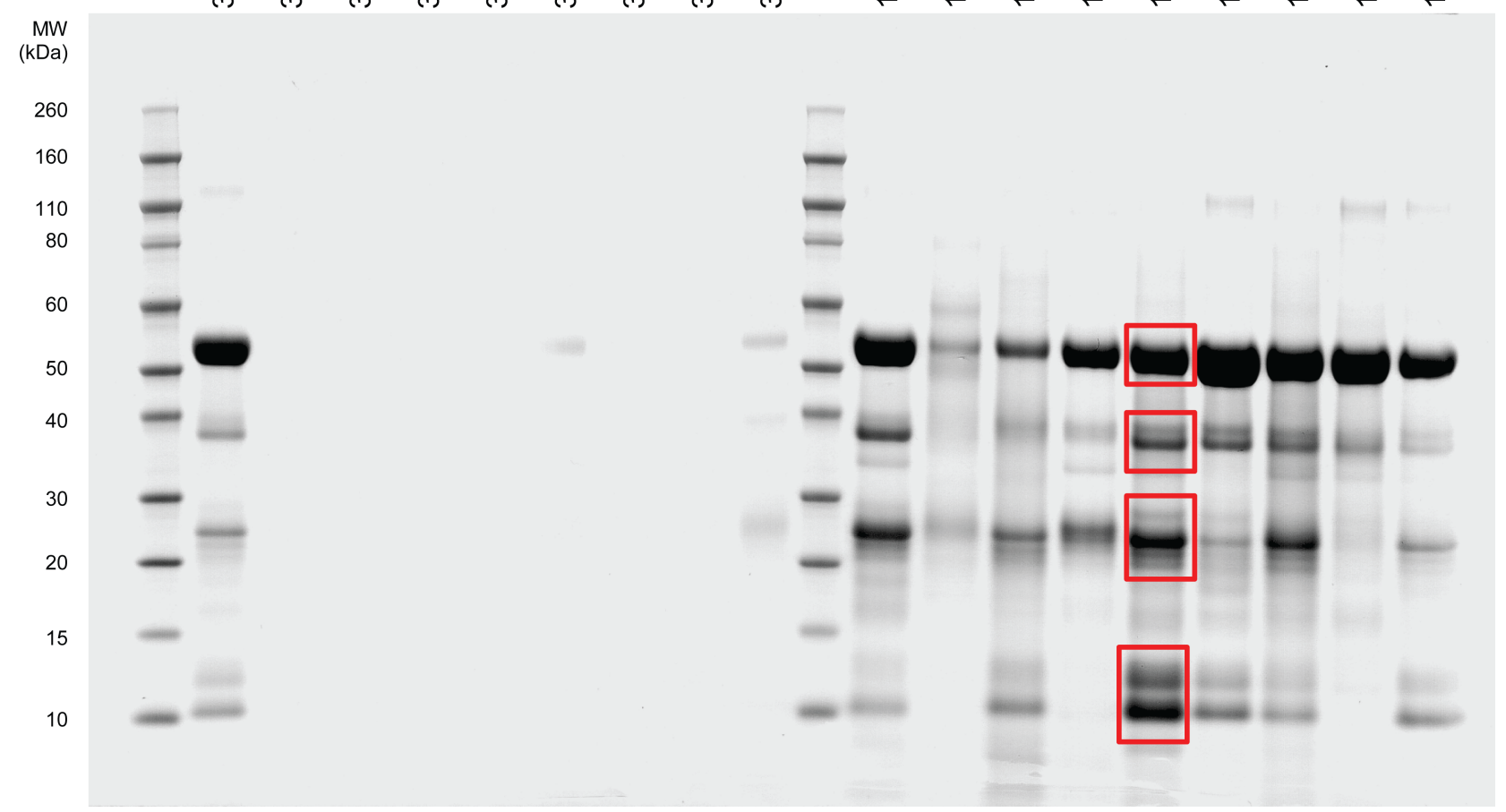

Figure 2 SDS-PAGE of benzamidine-Sepharose enriched fraction of faecal extract samples. SDS-PAGE of chromatographic fractions of faecal extracts from patients with irritable bowel syndrome with diarrhoea (IBS-D) and healthy volunteers. Individual reference numbers are shown and red boxes denote representative regions excised for component identification. Note that similar profiles of components were recovered for all diseased individuals and that little or no protein was detected in healthy volunteers except for one individual who showed an SDS-PAGE profile comparable with IBS-D patients. Retrospectively it was apparent she did experience pain and some urgency though not enough to meet Rome criteria. The average transit time of these nine IBS patients was $21 \mathrm{~h}$, stool frequency 3.0/day and urgency score 1.3 on a $0-3$ scale, which is typical for the group whose average values were $19 \mathrm{~h}$ for transit, stool frequency $2.6 /$ day and urgency score 1.6.

with a broad molecular size range (figure 2). As can be seen, several components were selectively retained and their relative abundance was increased in patients compared with healthy controls. Four representative molecular weight gel regions were excised and the human proteins $\alpha$-amylase $2 \mathrm{~A}$ and $2 \mathrm{~B}$, trypsin-1, -2 and -3 , chyomotrypsin-like elastase $3 \mathrm{~B}$, carboxypeptidase $B$ and $\alpha_{1}$-antitrypsin were identified (table 2) using proteomic procedures. A summary of the proteomics results is shown in online supplementary appendix table S2 and confirms the high specificity of the generated results, together with the high statistical significance and confidence in assigned identifications. There is inherently high homology between the various trypsin proteins, but multiple sequence alignment analyses (see online supplementary appendix figure S3) clearly show that there were data specific to each of the three gene products and also that autolytic fragments of the employed porcine trypsin sequencing proteinase did not contribute any spurious result. Tryptases have been implicated in IBS, and there are documented sequence homologies between trypsins and tryptases. However, multiple sequence alignments (see online supplementary appendix figure S4) clearly show that all detected peptide sequence results were uniquely specific to trypsins-that is, no primary sequence identified was common to tryptases.

\section{FP activity in patients with IBS-D versus healthy controls}

FP activity was measured in the first 36 patients with IBS-D and in 9 healthy controls. FP showed wide variability and was nonnormally distributed in patients with IBS-D (Shapiro-Wilks
$\mathrm{W}=0.8, \mathrm{p}=0.0003$ ) but not in healthy controls. Values were significantly increased in patients with IBS-D compared with healthy controls (median (IQR) 451 (61-963) vs 147 (IQR 82-336) units trypsin/mg protein, $\mathrm{p}=0.038$, Mann-Whitney $\mathrm{U}$ test).

\section{Faecal levels of elastase and amylase in patients with IBS-D versus controls}

Faecal elastase values were normally distributed but were not significantly different in patients with IBS-D and healthy controls (mean \pm SEM $2.4 \pm 0.2$ vs $2.5 \pm 0.4 \mu \mathrm{g} / \mathrm{mg}$ stool, $\mathrm{p}=0.85$, $\mathrm{n}=36$ ). Pancreatic amylase (figure 3 ) showed a biphasic distribution which was somewhat higher in patients with IBS than in healthy controls (median (IQR) $122.4(0.48-325.2)$ vs 17.0 (10.3-102.8) units/mL), but this did not achieve statistical significance $(\mathrm{p}=0.07)$.

However, there was a clear gap between the value of $27.3 \mu / \mathrm{L}$ and the next value of $105.1 \mu / \mathrm{L}$, suggesting two groups within the patients with IBS. If those with faecal amylase values $>27.3 \mu / \mathrm{L}$ were compared with those with lower values, they had significantly higher FP (634 (220-1322) vs 276 (111-485) trypsin units/mg protein, $\mathrm{p}=0.03 \mathrm{Mann}-$ Whitney $\mathrm{U}$ test) and greater anxiety scores (11.1 (1.1) vs 6.4 (0.8), $\mathrm{p}=0.006)$. Although transit was numerically shorter $(21.8$ (4.2) h vs 27.4 (4.1) h), this difference was not significant.

\section{Mast cell tryptase}

Using the Phadia Immunocap assay for mast cell tryptase, we found undetectable levels $(<0.1 \mu \mathrm{g} / \mathrm{L})$ in stool samples from 79 
Table 2 Major components identified in benzamidine-Sepharose enriched fractions of patient faeces extracts

\begin{tabular}{|c|c|c|}
\hline Protein name & Gene name(s) & $\begin{array}{l}\text { Accession } \\
\text { number }\end{array}$ \\
\hline Human pancreatric $\alpha$-amylase 2A & AMY2A & P04746 \\
\hline Human $\alpha$-amylase 2B & AMY2B & P19961 \\
\hline Human trypsin-1 (trypsinogen I) & TRY1, PRSS1 & P07477 \\
\hline Human trypsin-2 (trypsinogen II) & TRY2, PRSS1 & P07478 \\
\hline Human trypsin-3/4 (trypsinogen III/IV) & $\mathrm{TRY} 3 / 4, \mathrm{PRSS} 3 / 4$ & P35030 \\
\hline $\begin{array}{l}\text { Human chymotrypsin-like elastase } \\
\text { family member } 3 \mathrm{~B}\end{array}$ & CELA3B & P08861 \\
\hline Human carboxypeptidase B & CPB1 & P15086 \\
\hline Human $\alpha-1$-antitrypsin & SERPINA1 & P01009 \\
\hline
\end{tabular}

Alternative names are given where relevant and gene names and the UniProt database accession numbers are provided for information.

patients with IBS-D and 20/21 healthy controls, even though spiking the sample with pure human mast cell tryptase (MCT, Sigma-Aldrich, Warrington, UK) did show that it could be detected in stool at concentrations down to $5.5 \mu \mathrm{g} / \mathrm{L}$.

\section{Effect of colonic cleansing in healthy controls using MoviPrep}

Twenty-three subjects completed the trial. Both the double dose of $1 \mathrm{~L}$ and the single dose of $2 \mathrm{~L}$ MoviPrep induced pronounced loose watery stools and an average 31-fold fall in bacterial counts on day 2 (data not shown). FP activity in stool samples passed on day 2 (day after the purge) was significantly elevated, rising from a median (IQR) of 253 (140-426) trypsin units/mg at visit 1 to 1031 (435-2296) at visit 2, falling again to baseline at day 14 (258 (149-547)) and 236 (156-310) trypsin units/mg on day 28 (figure 4). One-way ANOVA showed a significant effect of time $(p=0.001)$, post hoc comparison showed significantly higher levels at visit 2 than at visit $1 \quad(\mathrm{p}=0.0007$ Wilcoxon test, $\mathrm{n}=23$ ).

\section{Correlation with transit in IBS-D}

Seventy-nine samples from the placebo arm of the ondansetron trial were matched with colonic transit measurements and 21 healthy, age- and sex- matched volunteers. As with the baseline

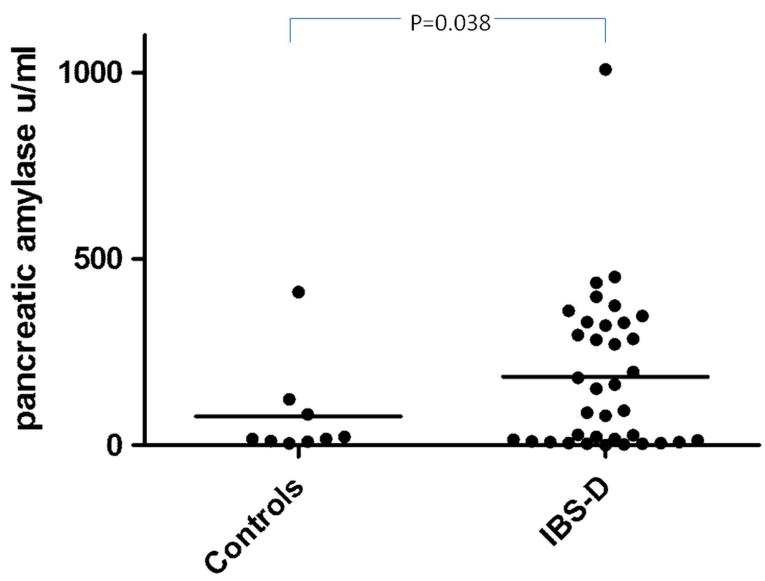

Figure 3 Faecal levels of amylase in patients with irritable bowel syndrome with diarrhoea (IBS-D) versus controls showing a biphasic distribution with one group of 22 showing a significant increase while a subgroup of 14 were not different from normal.

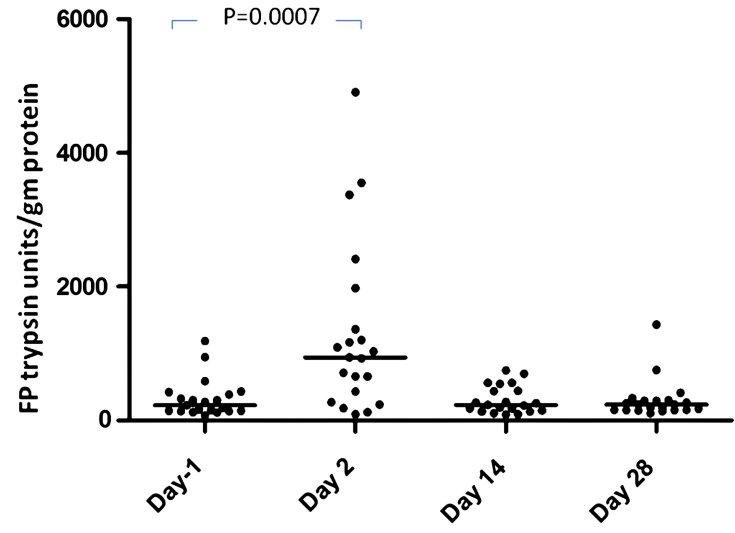

Figure 4 Faecal protease (FP) activity expressed in trypsin units/mg protein after colonic cleansing with MoviPrep at day -1 and days 2, 14 and 28 showing a significant rise in FSP activity after purging of colonic bacteria. One-way ANOVA for effect of time $(p=0.001$; day -1 vs day $2, \mathrm{p}=0.0007, \mathrm{n}=23$ ).

values of the first 36 patients, there was wide variability in FP levels in the larger group from the placebo arm and, although the values for patients with IBS were numerically greater than those for the matched healthy controls (median (IQR) 501 (245-1421) trypsin units/mg protein vs 302 (147-4) trypsin units/mg protein), the difference was not significant. If we combine all the healthy controls from parts 1 and 2, the control FP value is $302(147-475)$ trypsin units/mg protein $(n=51)$ and the difference from IBS-D is significant $(p=0.003)$ but, as figure 5 shows, there is a wide variation in both groups. In order to understand this variation we correlated transit and FP activities measured in patients with IBS-D on placebo which showed a significant negative correlation (Spearman $\mathrm{r}=-0.32(95 \% \mathrm{CI}$ -0.51 to 0.09 ), $p=0.005, n=79$, figure 6). A similar result was also found in healthy controls for whom we had transit measurements (Spearman $\mathrm{r}=-0.55 \quad(95 \% \mathrm{CI}-0.81$ to 0.11 ), $\mathrm{p}=0.014, \mathrm{n}=20$ ). FP activity also correlated significantly with the average urgency in patients with IBS patients (Spearman $\mathrm{r}=0.26(95 \%$ CI 0.03 to 0.47$), \mathrm{p}=0.02, \mathrm{n}=73$, figure 7$)$.

There were no correlations with the average daily pain score and stool frequency ( $p=0.18$ and 0.75 , respectively). However, there was a significant correlation with anxiety $(r=0.30$, $\mathrm{p}=0.01)$, depression $(\mathrm{r}=0.3, \mathrm{p}=0.01)$ and psychological distress $(r=0.3, p=0.01)$. These psychological variables are closely

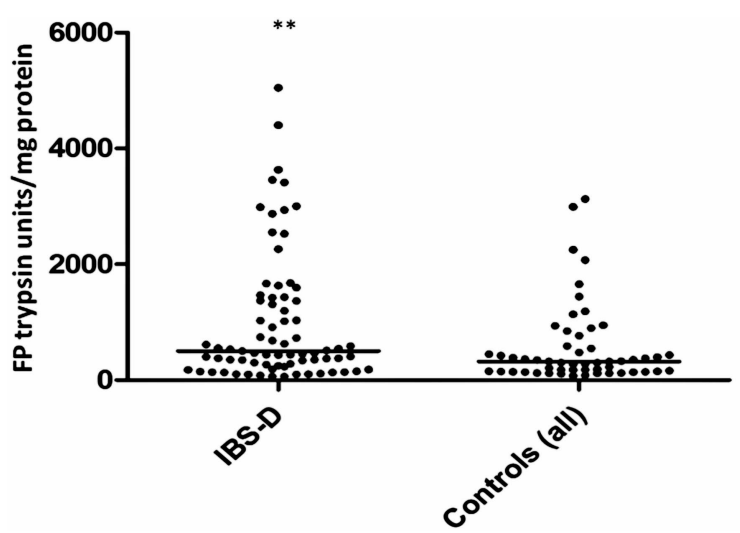

Figure 5 Faecal protease activity expressed in trypsin units/mg protein showing that patients with irritable bowel syndrome with diarrhoea had significantly higher values than controls, but with wide variability in both groups. ${ }^{* *} \mathrm{p}=0.003$ (Mann-Whitney $U$ test). 


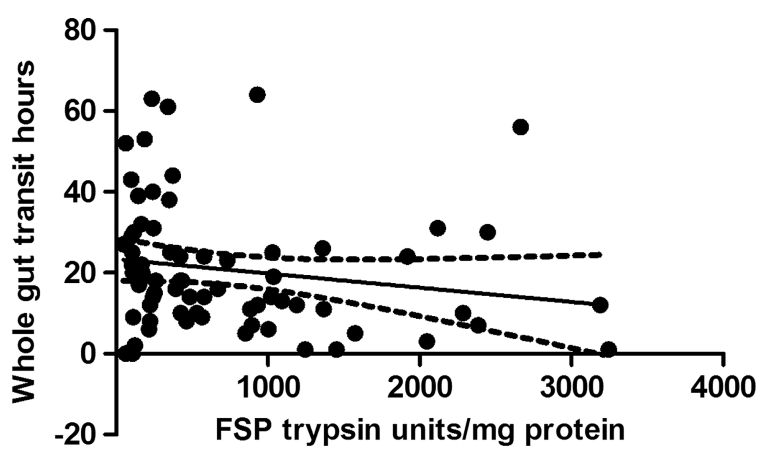

Figure 6 Inverse correlation between faecal serine protease (FSP) activity and whole gut transit in patients with irritable bowel syndrome with diarrhoea (Spearman $r=-0.32, p=0.005, n=79$ ).

correlated and, when entered with urgency into a backwards logistic regression model using the 72 cases with data for all variables, only depression remained an independent predictor in the model $(p=0.012)$. Ondansetron slowed transit, increasing transit time while decreasing FP with a negative correlation between the rise in transit and the increase in FP $(r=-0.40$, $\mathrm{p}=0.003$ ) in the 55 patients with complete values for both FP and transit at both time points.

\section{DISCUSSION}

We have confirmed previous reports ${ }^{2}$ that FSP activity is increased in some patients with IBS-D, but we also show that there is considerable overlap with normal subjects without IBS. We increased our understanding of this phenomenon by characterising the proteins responsible for the serine protease activity, showing that most of this activity is probably due to human pancreatic enzymes.

While the pancreas is the most likely source of trypsin-like activity, enterocytes also contain trypsin mRNA and trypsin-like immunoreactivity, ${ }^{14}$ although the amounts are small relative to expression in the pancreas. However, as has recently been shown, trypsin levels in colonic biopsies are increased in IBS-D, ${ }^{15}$ so we cannot be certain how much of the observed increase in FP is from enterocytes and how much is pancreatic. Furthermore, human epithelial cell lines secrete trypsinogen $\mathrm{IV}^{16}$ the mRNA of which has also been found to be increased 1.5 -fold in human small intestinal biopsies from patients with IBS-D. ${ }^{17}$ Trypsinogen IV is known to activate PAR-2 receptors ${ }^{16}$

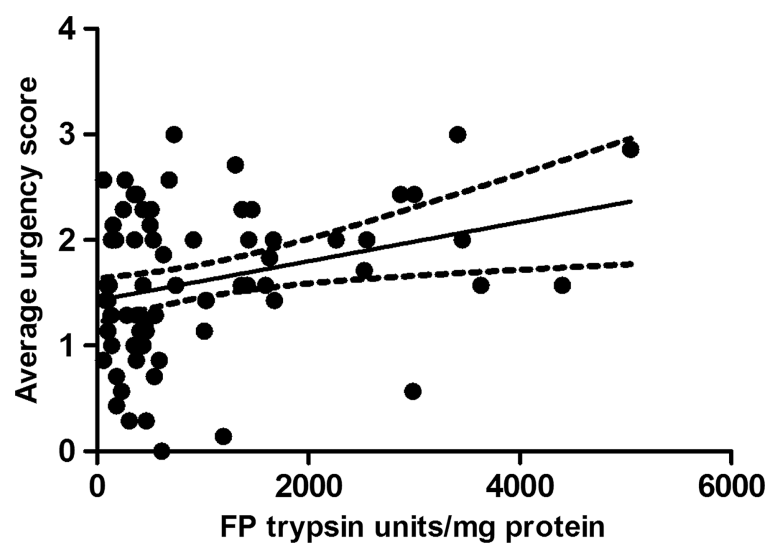

Figure 7 Positive correlation between faecal protease (FP) activity and days/week with urgency in patients with irritable bowel syndrome with diarrhoea (Spearman $r=0.26, p=0.02, n=73$ ). and so may be important in activating afferent nerves, inflammatory changes and increased permeability, all of which could contribute to IBS symptoms.

Using a benzamindine affinity resin, we extracted FSPs and characterised them by mass spectrometric analysis of proteolysed components in gel electrophoresis features and identified several human enzymes including amylase, elastase, carboxypeptidase and trypsin. However, it should be noted that, of these enzymes, only trypsin is known to activate the PAR-2 receptor and hence would be predicted to influence visceral sensitivity, gut barrier function and immune function. Surprisingly, using a highly specific immunoassay we found that endogenous mast cell tryptase was undetectable in faecal extracts.

Several important papers have examined the release of trypsin-like mediators from supernatants of IBS biopsies and most $^{15} 18{ }^{19}$ but not all ${ }^{20}$ have found increased release. Others have suggested that FSPs might be of bacterial origin, but we found major components in purified faecal extract to be human derived and values of FP rose when bacterial counts were depleted by MoviPrep, suggesting that any bacterial contribution to FP is small. Earlier studies argued that, because high levels of FP activity were not seen in bacterial gastroenteritis, this meant that fast transit was not important. ${ }^{2}$ However, we believe our study is better controlled to study just the effect of fast transit without other factors which may well be responsible for the lack of rise in gastroenteritis. Thus, most patients with gastroenteritis become anorexic, which would be expected to reduce pancreatic enzyme secretion, while our subjects ate a $400 \mathrm{kcal}$ low residue diet the night before the study which would be expected adequately to stimulate pancreatic secretion.

Since our data suggested a probable pancreatic origin for the serine protease activity, we examined other pancreatic enzymes in the stool samples. Faecal amylase was assessed using a specific immunoassay to identify human pancreatic amylase and showed a similar trend to FP with increased values which correlated with FP activity. The amylase levels suggested two groups of patients, and we found that patients with IBS-D with higher faecal amylase had significantly higher FP activity and greater anxiety scores and a tendency to faster transit than those with lower values, suggesting that these increases might both be due to accelerated transit. Previous studies of faecal trypsin indicate that the very substantial amounts of pancreatic trypsin secreted each day are mostly degraded during transit through the colon since the $24 \mathrm{~h}$ faecal output is approximately $1 \mathrm{mg}$ compared with an ileostomy output of 50-200 mg. ${ }^{21}$ Antibiotic therapy which inhibits bacterial degradation increases faecal trypsin and elastase in both rats $^{22}$ and humans. ${ }^{23}$ The pancreas is the major source of human faecal elastase since very little is excreted in the faeces of patients with pancreatic insufficiency as assessed by intubated pancreatic function testing. ${ }^{24}$ Elastase appears to be inherently more resistant to bacterial degradation. Thus, after reducing the faecal bacterial content in humans by oral antibiotics, trypsin levels rose 100-fold while elastase levels rose only $2-3$-fold. ${ }^{21}$ This may explain why elastase is not increased in IBS-D as little degradation occurs, so faecal levels reflect pancreatic secretion which is not expected to be different in patients with IBS-D and controls.

We therefore hypothesised that accelerating transit would increase FP activity and, furthermore, that faster transit in IBS-D would predict higher FP activity. We tested this as part of our RCT of ondansetron and were able to show a negative correlation of FP activity with whole gut transit in IBS-D as predicted.

Purging the colon and substantially reducing bacterial counts using MoviPrep increased FP activity in the stools of healthy 
volunteers to levels seen in some patients with IBS-D, supporting our hypothesis that raised levels in IBS-D may well reflect a reduction in bacterial degradation of endogenous pancreatic trypsin secondary to either accelerated transit and/or altered colonic microbiota.

The increase in FP activity due to undegraded pancreatic enzymes may well be important since trypsin is a potent activator of PAR-2 receptors. This activation can increase gut permeability $^{2},{ }^{25}$ which, in some animal ${ }^{26} 27$ and human studies, is very clearly linked to abdominal pain and visceral hypersensitivity. ${ }^{28}$ Furthermore, sensitising the distal gut may aggravate diarrhoea and urgency. Slowing transit by any means would be predicted to reduce faecal tryptic activity and benefit symptoms. The correlation of FP activity with urgency also suggests that new therapies targeting FP could be useful in the treatment of this most debilitating symptom of IBS-D. Finally, if these changes in FP are due to changes in the ability of gut microbiota to degrade protease, it opens up the possibility that modifying the microbiota by diet or probiotics might benefit IBS symptoms by reducing FP.

Acknowledgements We would like to thank Mr M Carlile and Mr K Bailey (School of Biomedical Sciences, University of Nottingham) for technical assistance with both affinity chromatography and protein analysis procedures and Simon Martin (Department of Immunology, Nottingham University Hospitals NHS Trust) for doing the immunoassays for mast cell tryptase.

Contributors Conception, design and overall supervision of study: RS, GS, DT, PW and KG. Collection of samples: KG, CL, RB, MH and ML. Analysis of samples: GS, $\mathrm{DT}, \mathrm{RB}, \mathrm{ML}$ and RL. Write up: All authors.

Funding The study involved samples from several studies including the ondansetron trial funded by the NIHR Research for Patient Benefit programme and the MoviPrep study, an investigator initiated study funded by Norgine. The assay development was funded by Lesaffre International as part of another project. The funders have reviewed the document but were not involved in the analysis of the samples nor the write-up of the study. The Nottingham Biomedical Research Unit is supported by a grant from the National Institute for Health Research (NIHR). RS is supported by an NIHR Senior Investigator Award. This paper presents independent research funded by the NIHR. The views expressed are those of the authors and not necessarily those of the NHS, the NIHR or the Department of Health.

Competing interests RS: grant support from Lesaffre and Norgine; advisory board member for Almirral, Shire and Ironwood; lecturing fees from Abbott and Shire. PW: grant support form Danone; advisory board member for Almirral, Shire, Danone and Norgine; lecturing fees from Abbott, Danone and Shire. The other authors have no competing interests.

Ethics approval The patient studies were approved by the National Research Ethics Committee East Midlands-Nottingham 2 and the healthy volunteer studies by the University of Nottingham Medical School Research Ethics Committee.

Provenance and peer review Not commissioned; externally peer reviewed.

Data sharing statement We include an online appendix with extra data.

Open Access This is an Open Access article distributed in accordance with the Creative Commons Attribution Non Commercial (CC BY-NC 3.0) license, which permits others to distribute, remix, adapt, build upon this work non-commercially, and license their derivative works on different terms, provided the original work is properly cited and the use is non-commercial. See: http://creativecommons.org/licenses/by-nc/3.0/

\section{REFERENCES}

1 Spiller R, Aziz Q, Creed F, et al. Guidelines on the irritable bowel syndrome: mechanisms and practical management. Gut 2007:56:1770-98.

2 Gecse K, Roka R, Ferrier L, et al. Increased faecal serine protease activity in diarrhoeic IBS patients: a colonic lumenal factor impairing colonic permeability and sensitivity. Gut 2008;57:591-9.
3 Mertz $\mathrm{H}$, Naliboff B, Munakata J, et al. Altered rectal perception is a biological marker of patients with irritable bowel syndrome. Gastroenterology 1995;109: 40-52.

4 Roka R, Rosztoczy A, Leveque $M$, et al. A pilot study of fecal serine-protease activity: a pathophysiologic factor in diarrhea-predominant irritable bowel syndrome. Clin Gastroenterol Hepatol 2007;5:550-5.

5 Otten CM, Kok L, Witteman BJ, et al. Diagnostic performance of rapid tests for detection of fecal calprotectin and lactoferrin and their ability to discriminate inflammatory from irritable bowel syndrome. Clin Chem Lab Med 2008;46:1275-80.

6 Garsed K, Singh G, Tooth D, et al. Origin of increased fecal serine protease in patients with irritable bowel syndrome and diarrhoea (IBS-D). Gut 2011;60:A25.

7 MacFarlane GT, Allison C, Gibson SA, et al. Contribution of the microflora to proteolysis in the human large intestine. J App/ Bacteriol 1988;64:37-46.

8 Garsed K, Hastings M, Marciani L, et al. Ondansetron is an effective treatment for patients with diarrhoea predominant irritable bowel syndrome. Gut 2011;60(Suppl 3):A40.

9 Garsed KC, Marciani L, Fields A, et al. Mode of action of a macrogol formulation on distribution of intestinal fluid: a MRI study. Gastroenterology 2012;142:S814.

10 Metcalf AM, Phillips SF, Zinsmeister AR, et al. Simplified assessment of segmental colonic transit. Gastroenterology 1987;92:40-7.

11 Bradford M. A rapid and sensitive method for the quantitation of microgram quantities of protein utilizing the principle of protein-dye binding. Anal Biochem 1976;72:248-54.

12 Tooth DJ, Krishna VG, Layfield R. An economical high-throughput protocol for multidimensional fractionation of proteins. International Journal of Proteomics 2012;2012:735132.

13 Hellman U, Wernstedt C, Gonez J, et al. Improvement of an "In-Gel" digestion procedure for the micropreparation of internal protein fragments for amino acid sequencing. Anal Biochem 1995;224:451-5.

14 Koshikawa N, Hasegawa S, Nagashima Y, et al. Expression of trypsin by epithelial cells of various tissues, leukocytes, and neurons in human and mouse. Am J Pathol 1998:153:937-44.

15 Cenac N, Andrews CN, Holzhausen $\mathrm{M}$, et al. Role for protease activity in visceral pain in irritable bowel syndrome. J Clin Invest 2007:117:636-47.

16 Cottrell GS, Amadesi S, Grady EF, et al. Trypsin IV, a novel agonist of protease-activated receptors 2 and 4. J Biol Chem 2004;279:13532-9.

17 Kerckhoffs AP, Ter Linde JJ, Akkermans LM, etal. Trypsinogen IV, serotonin transporter transcriptlevels and serotonin content are increased in small intestine of irritablebowe syndrome patients. Neurogastroentero/Motil 2008;20:900-7.

18 Barbara G, Wang B, Stanghellini V, et al. Mast cell-dependent excitation of visceral-nociceptive sensory neurons in irritable bowel syndrome. Gastroenterology 2007;132:26-37

19 Buhner S, Li Q, Vignali S, et al. Activation of human enteric neurons by supernatants of colonic biopsy specimens from patients with irritable bowel syndrome. Gastroenterology 2009;137:1425-34.

20 Klooker TK, Braak B, Koopman KE, et al. The mast cell stabiliser ketotifen decreases visceral hypersensitivity and improves intestinal symptoms in patients with irritable bowel syndrome. Gut 2010;59:1213-21.

21 Bohe M, Borgstrom A, Genell S, et al. Determination of immunoreactive trypsin pancreatic elastase and chymotrypsin in extracts of human feces and ileostomy drainage. Digestion 1983;27:8-15.

22 Genell S, Gustafsson BE. Impaired enteric degradation of pancreatic endopeptidases in antibiotic-treated rats. Scand J Gastroenterol 1977;12:801-9.

23 Borgstrom A, Genell S, Ohlsson K. Elevated fecal levels of endogenous pancreatic endopeptidases after antibiotic treatment. Scand J Gastroenterol 1977;12:525-9.

24 Goldberg DM. Proteases in the evaluation of pancreatic function and pancreatic disease. Clin Chim Acta 2000;291:201-21.

25 Cenac N, Coelho AM, Nguyen C, et al. Induction of intestinal inflammation in mouse by activation of proteinase-activated receptor-2. Am J Pathol 2002;161:1903-15.

26 It-Belgnaoui A, Bradesi S, Fioramonti J, et al. Acute stress-induced hypersensitivity to colonic distension depends upon increase in paracellular permeability: role of myosin light chain kinase. Pain 2005;113:141-7.

27 Gareau MG, Jury J, Perdue MH. Neonatal maternal separation of rat pups results in abnormal cholinergic regulation of epithelial permeability. Am J Physiol Gastrointest Liver Physiol 2007;293:G198-203.

28 Zhou Q, Zhang B, Verne GN. Intestinal membrane permeability and hypersensitivity in the irritable bowel syndrome. Pain 2009;146:41-6. 